\title{
Robot-assisted real-time magnetic resonance image-guided transcatheter aortic valve replacement
}

\author{
Justin G. Miller, MD, Ming Li, PhD, Dumitru Mazilu, PhD, Tim Hunt, BS, and Keith A. Horvath, MD
}

\begin{abstract}
Background: Real-time magnetic resonance imaging (rtMRI)-guided transcatheter aortic valve replacement (TAVR) offers improved visualization, real-time imaging, and pinpoint accuracy with device delivery. Unfortunately, performing a TAVR in a MRI scanner can be a difficult task owing to limited space and an awkward working environment. Our solution was to design a MRI-compatible robot-assisted device to insert and deploy a self-expanding valve from a remote computer console. We present our preliminary results in a swine model.
\end{abstract}

Methods: We used an MRI-compatible robotic arm and developed a valve delivery module. A 12-mm trocar was inserted in the apex of the heart via a subxiphoid incision. The delivery device and nitinol stented prosthesis were mounted on the robot. Two continuous real-time imaging planes provided a virtual real-time 3-dimensional reconstruction. The valve was deployed remotely by the surgeon via a graphic user interface.

Results: In this acute nonsurvival study, 8 swine underwent robot-assisted rtMRI TAVR for evaluation of feasibility. Device deployment took a mean of $61 \pm 5$ seconds. Postdeployment necropsy was performed to confirm correlations between imaging and actual valve positions.

Conclusions: These results demonstrate the feasibility of robotic-assisted TAVR using rtMRI guidance. This approach may eliminate some of the challenges of performing a procedure while working inside of an MRI scanner, and may improve the success of TAVR. It provides superior visualization during the insertion process, pinpoint accuracy of deployment, and, potentially, communication between the imaging device and the robotic module to prevent incorrect or misaligned deployment. (J Thorac Cardiovasc Surg 2016;151:1407-12)

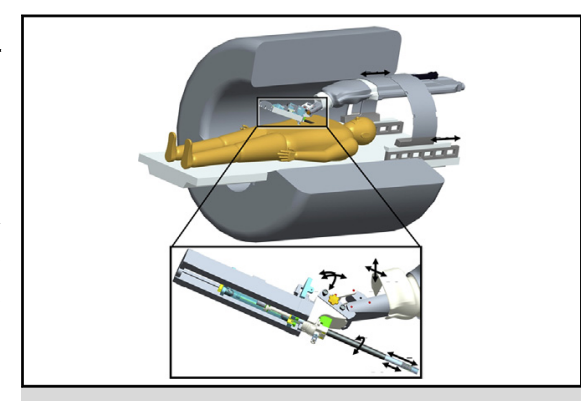

Valve delivery module and robot platform in the MRI bore with a patient.

Central Message

We show the feasibility of robot-assisted rtMRI-guided TAVR, which has benefits over current imaging and delivery technologies.

\section{Perspective}

Real-time MRI-guided TAVR overcomes the limitations of currently available imaging modalities; however, performing TAVR in an MRI scanner can be difficult, owing to limited space. We designed a robot-assisted device to deploy a self-expanding valve. This method provides superior visualization and deployment with pinpoint accuracy, and can prevent misaligned deployment.

See Editorial Commentary page 1413.
Aortic stenosis is the most common type of valvular heart disease in the United States. ${ }^{1-3}$ This disease process has a long latency period; however, patients rapidly decline after becoming symptomatic. Previously a patient's only chance for substantially prolonging survival was to undergo cardiopulmonary bypass with surgical aortic valve replacement. ${ }^{4-6}$ Unfortunately, some of these patients were inappropriate or high-risk surgical candidates. ${ }^{7}$ Since its approval by the Food and Drug Administration in 2011,

\footnotetext{
From the Cardiothoracic Surgery Research Program, National Heart, Lung and Blood Institute, National Institutes of Health, Bethesda, Md.

This research received intramural funding from the National Heart, Lung and Blood Institute.

Received for publication July 23, 2015; revisions received Nov 23, 2015; accepted for publication Nov 25, 2015; available ahead of print Jan 14, 2016.

Address for reprints: Keith A. Horvath, MD, 10 Center Dr, Bldg 10, Rm B1D47, Bethesda, MD 20892 (E-mail: horvathka@mail.nih.gov).

$0022-5223 / \$ 36.00$

Copyright (c) 2016 by The American Association for Thoracic Surgery

http://dx.doi.org/10.1016/j.jtcvs.2015.11.047
}

transcatheter aortic valve replacement (TAVR) has become a viable treatment option for patients with otherwise inoperable or high-risk aortic stenosis. ${ }^{7,8}$

Although there have been multiple advances in valve development and valve delivery technology, the imaging modality has remained unchanged. Currently TAVR is performed with a combination of fluoroscopy and transesophageal echocardiography (TEE), which has multiple limitations. The TAVR procedure currently necessitates various imaging modalities for different stages of the procedure. . $^{90}$ Preprocedural imaging usually includes echocardiography in combination with multidetector computed tomography (MDCT) or computed tomography (CT) angiography.

Scanning this QR code will take you to the article title page.

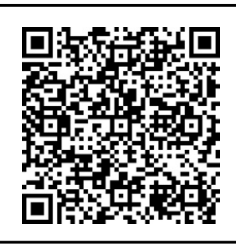




\section{Abbreviations and Acronyms}

$\mathrm{CT}=$ computed tomography

MDCT $=$ multidetector computed tomography

rtMRI = real-time magnetic resonance imaging

TAVR $=$ transcatheter aortic valve replacement

TEE $=$ transesophageal echocardiography

Postprocedural imaging also routinely uses a combination of fluoroscopy and TEE to confirm valve placement and cardiac function. ${ }^{10-13}$ Fluoroscopy has multiple limitations, including poor soft tissue contrast, a requirement for rapid ventricular pacing, radiation exposure to the patient and surgical team, and a risk of contrast-induced nephropathy. ${ }^{14-17}$ Real-time magnetic resonance imaging (rtMRI) guidance overcomes these limitations with improved soft tissue contrast in addition to a 3-dimensional visualization of the anatomic structures. rtMRI has the added benefit of completing the preprocedural, intraprocedural, and postprocedural imaging with a single device.

rtMRI guidance has been proposed as the future of TAVR. ${ }^{16,18,19}$ Unfortunately, performing a TAVR procedure while working in the bore of an MRI scanner can be a difficult task, owing to limited space and a potentially awkward working environment. Our solution to this problem was to design an MRI-compatible robot-assisted device capable of inserting and deploying a self-expanding valve from a remote computer console. Here we present our preliminary results using this device to perform transapical TAVR in a swine model.

\section{MATERIALS AND METHODS MRI System}

The MRI system comprises several components, including a 1.5-T MRI unit, interactive image reconstruction software, and advanced pulse-sequence technology. ${ }^{20-22}$ A MAGNETOM Aera MRI system (Siemens Medical Solutions, Munich, Germany) was used for this experiment. This device's bore size of $145-\mathrm{cm}$ long $\times 70$ - $\mathrm{cm}$ wide provides adequate clearance above the patient for the robot system. The system can generate high-quality images at 5-10 frames per second with low latency for fully interactive, real-time imaging; however, in this study, standard imaging sequences were used for preprocedural planning and postprocedural assessment. Interactive Front End navigation software (Siemens Corporate Research, Munich, Germany), along with an interactive real-time pulse sequence (BEAT_IRTTT), provided real-time navigation during valve deployment. The Interactive Front End navigation software obtains multiple slices in rapid succession that can be displayed simultaneously to provide a 3-dimensional rendering. The software allows rapid adjustment of the imaging planes to allow for real-time device tracking. ${ }^{20-24}$

\section{Self-Expanding Stent and Delivery Device}

A self-expanding nitinol stent was designed and engineered for this experiment (Figure 1). ${ }^{25,26}$ Although several nitinol stents are commercially available, including the CoreValve and SAPIEN valve, we designed our own stent for this experiment. While the geometry of some
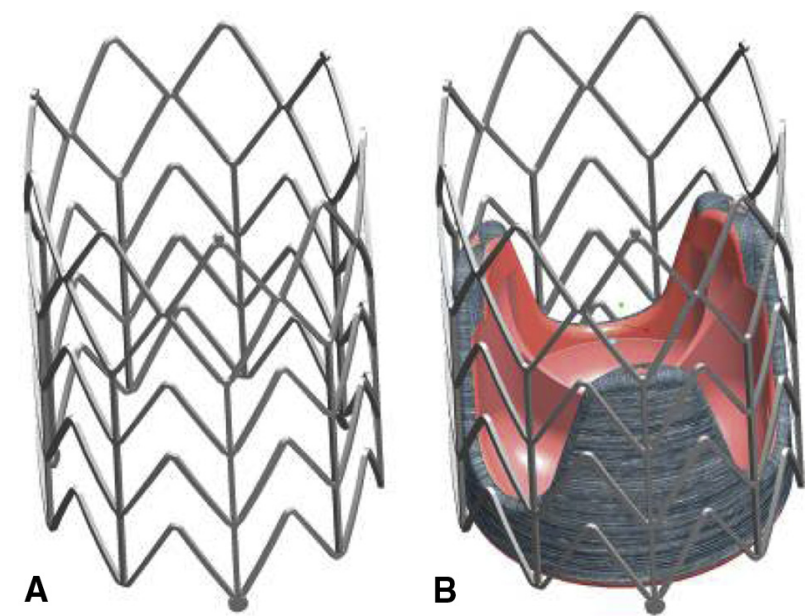

FIGURE 1. A, Self-expanding stent. B, Bioprosthetic valve affixed in a self-expanding stent.

stents uses a diamond cell shape, this design may cause increased stress along the stent during the crimping process. Our stent design is based on a chevron shape, which minimizes stress along the chevron cell shape. The chevron shape also prevents stent migration, owing to the selfanchoring properties of the pointed ends of the chevron. The stent is laser-cut from a biocompatible nitinol tube to an expanded diameter of $26 \mathrm{~mm}$ and a length of $35 \mathrm{~mm} .^{27}$ The stent is then compressed with a custom-made crimping device and inserted into the delivery device. ${ }^{17}$ The stent expands to its open configuration on release from the delivery system.

We also developed a delivery device for inserting and deploying the stented prosthesis. This delivery device consists of an inner rod and an outer sheath, which are controlled by the robotic delivery module's pneumatic actuators. The outer sheath also protects the nitinol stent before deployment. The delivery device is MRI-compatible and fits into a 12-mm trocar. ${ }^{27}$

\section{Robotic Assistance System}

We developed an MRI-compatible robotic surgical assistant system to deliver the aortic valve prostheses. ${ }^{28,29}$ This system consists of a positioning module, a valve delivery module, custom-designed software, and a graphical user interface. The positioning module consists of a modified Innomotion MRI-compatible robotic arm (Innomedic, Herxheim, Germany). The positioning module holds the valve delivery module and adjusts the planned trajectory of the valve delivery device. The positioning module was modified to hold and manipulate the valve delivery module and has 5 degrees of freedom. Movements include axial, vertical, and horizontal translation, as well as pitch and yaw. The valve delivery module has 3 degrees of freedom, which includes roll, translation, and insertion of the delivery device. The valve delivery module was designed to work in conjunction with the robotic arm to insert and deploy the stent. ${ }^{22}$ The combination of the movements between the positioning module and valve delivery module include all of the movements that a human would use when inserting and deploying the stent.

To maintain image quality and prevent heat transfer to the patient, the valve delivery module is made of nonconductive plastic materials, pneumatic actuators, and magnetotranslucent fiber-optical encoders. The profile of the valve delivery module and positioning module allow the system to fit into a standard closed MRI scanner (Figure 2). The primary control computer uses a custom software program to control the robotic system via an optic network. For precision with valve deployment, the 


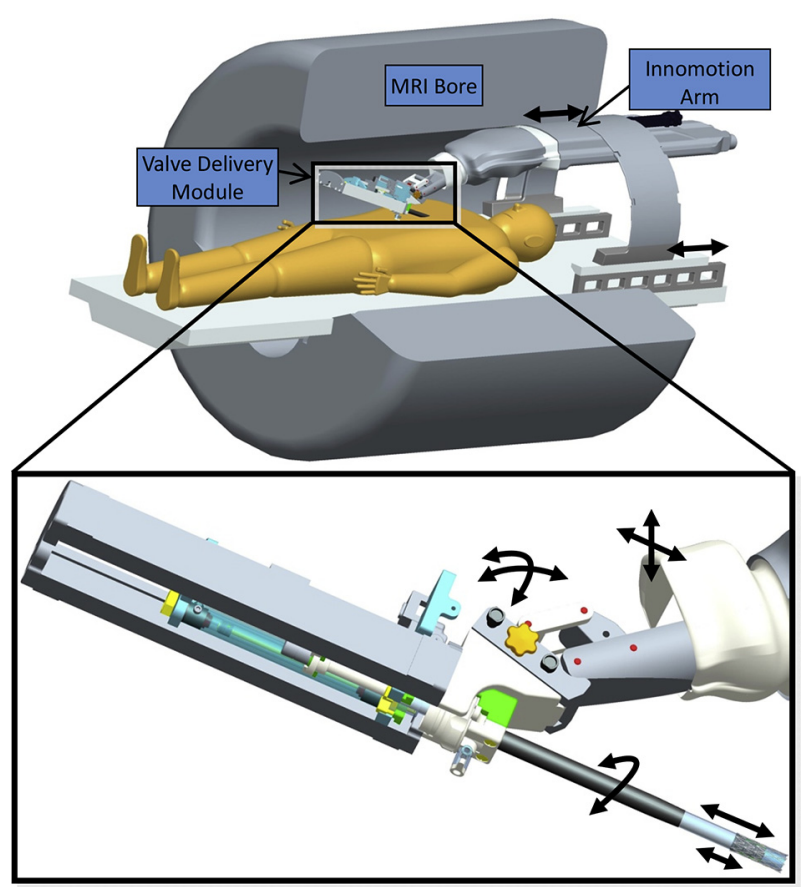

FIGURE 2. Valve delivery module and robot platform in the MRI bore with a patient. MRI, Magnetic resonance imaging.

accuracy of the linear joints is $\sim 1 \mathrm{~mm}$, and the accuracy of the rotational joints is within $1^{\circ} .^{30}$ The interactive control interface allows the user to plot the next move for the delivery device, such as increasing angulation or advancing the delivery device. While the robot is executing a maneuver, the user can watch the device in real time. If any movement needs to be modified, the user can start, stop, change, or resume the delivery device motion at any time.

\section{Animal Surgery Protocol}

All experiments were performed in accordance with protocols approved by the National Institutes of Health's Animal Care and Use Committee. Induction included intramuscular injections of midazolam $(0.5 \mathrm{mg} / \mathrm{kg})$ and ketamine $(25 \mathrm{mg} / \mathrm{kg})$. After induction, the animals were intubated and then maintained on mechanical ventilation with isoflurane $(0.5 \%$ $2.5 \%$ ). The animals' end tidal $\mathrm{CO}_{2}, \mathrm{O}_{2}$ saturation, arterial blood pressure, and electrocardiographic telemetry were monitored throughout the entire procedure. Body temperature was maintained with a forced-air warming blanket. Before the start of the procedure, the animals received intravenous amiodarone (150-300 mg) to treat arrhythmias, and before trocar insertion they received anticoagulation with heparin $(300 \mathrm{U} / \mathrm{kg})$. After completion of the experiment, the fully anesthetized animals were euthanized with an intravenous injection of phenobarbital $(150 \mathrm{mg} / \mathrm{kg})$. The times and details of the procedures were recorded.

\section{Valve Deployment}

Eight swine weighing between 45 and $55 \mathrm{~kg}$ underwent robot-assisted rtMRI-guided TAVR. Each animal was sedated and intubated, then prepped and draped in a supine position. A 6-cm subxiphoid incision was made, and the pericardium was opened. Two concentric purse string sutures were placed around the apex of the heart. An incision was made in the apex, and a $12-\mathrm{mm}$ trocar was inserted. The animal was then transferred to the MRI machine, where a surface radiofrequency coil was secured to the anterior chest to enhance signal reception. The delivery device was loaded

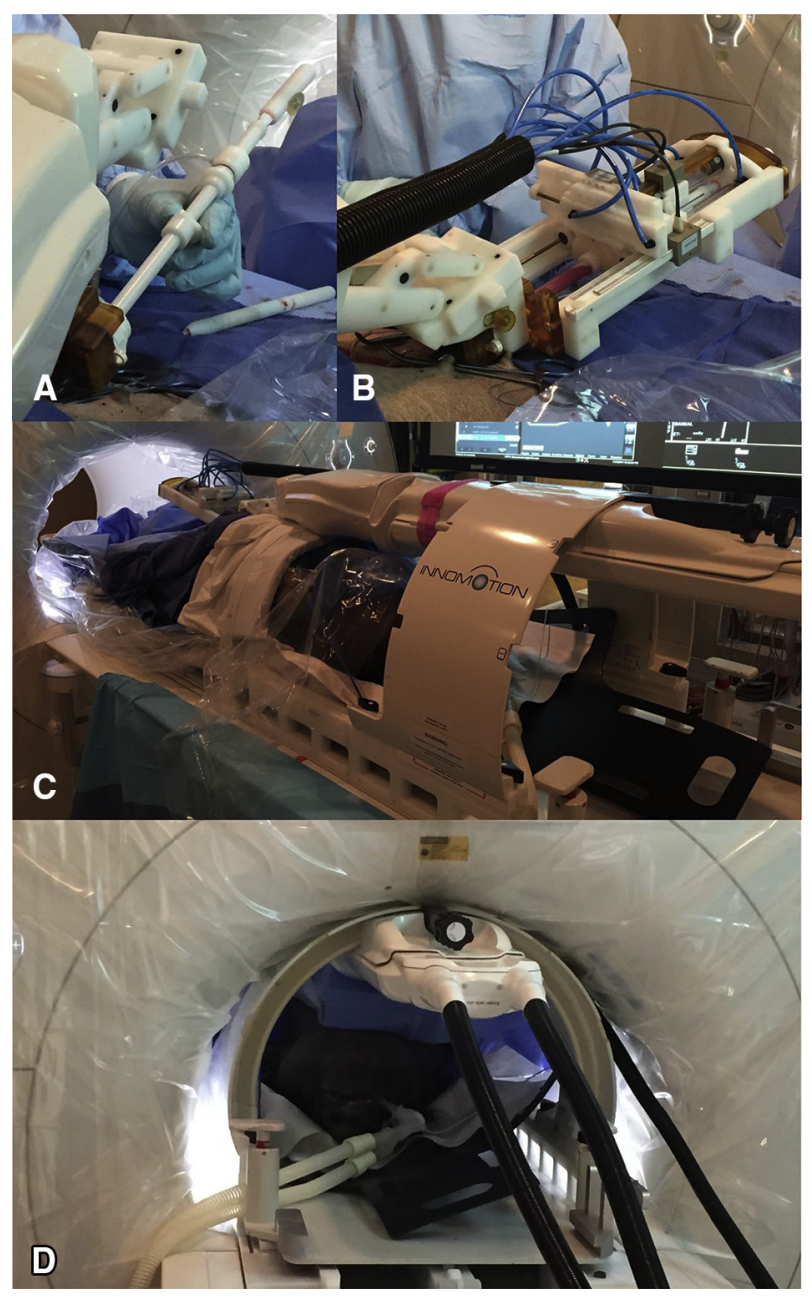

FIGURE 3. A, Insertion of the delivery device and nitinol stent. B, Valve delivery module mounted on a robotic arm. C, Patient and robot platform. D, Patient and robot platform in an MRI scanner.

with the stent, and the stent and delivery device were inserted into the opening of the trocar (Figure 3,A). The positioning module and valve delivery module were then affixed to the MRI table, and the delivery device was attached to the valve delivery module (Figure 3, $B$ and $C$ ). The MRI table with the robot and animal were then moved into the bore (Figure 3, D).

A predeployment scan was performed to confirm annulus and aortic root size and to allow the physician to plan the trajectory of the delivery device. For deployment, 2 imaging planes (long-axis and short-axis views) were used to create a virtual real-time 3-dimensional reconstruction. The physician was able to adjust the delivery device during insertion and to deploy the stent with the robot system via the graphical user interface and software program. The axial slices were shifted as needed to visualize the delivery device and maintain proper orientation for stent delivery.

Under rtMRI guidance, the delivery device was advanced until it was across the aortic valve (Figure $4, A$ ). Once the delivery device reached the proper position, the device was slowly deployed (Figure 4, B). When the stent was partially deployed, the physician could reposition the valve as needed to ensure correct and precise placement. Once the valve was deployed (Figure $4, C$ ), the delivery catheter system was withdrawn from the trocar (Figure 4,D). Postdeployment images were acquired to confirm 


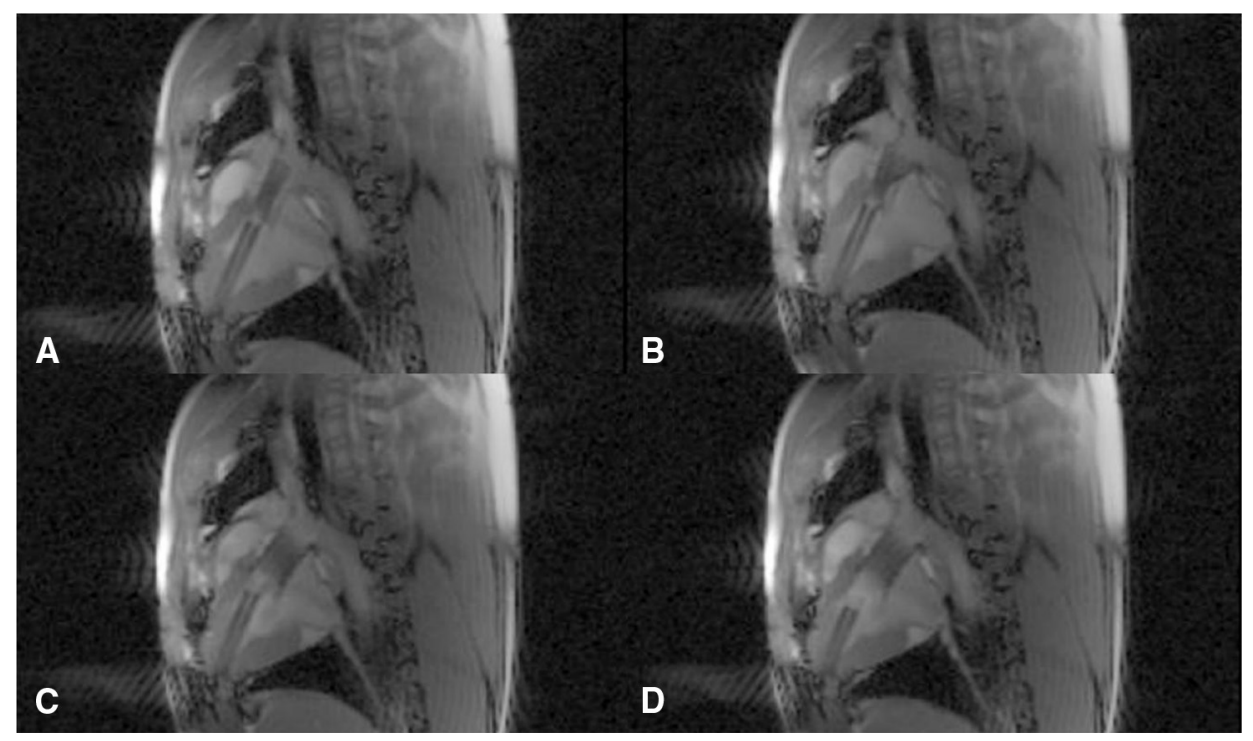

FIGURE 4. Valve deployment from the delivery device. A, Delivery device advanced across the aortic valve. B, Sheath retracting to deploy the valve. C, Valve deployed. D, Delivery device backing out.

the position of the prostheses. Gated CINE-MRI was used to assess valve placement. Necropsy was performed on all animals after valve placement to grossly assess the precision of placement. ${ }^{16,18,20,22}$

\section{RESULTS}

In this acute nonsurvival study, 8 swine underwent successful robot-assisted rtMRI TAVR for evaluation of feasibility. The subxiphoid incision with trocar placement took a mean time of $18 \pm 6$ minutes. The predeployment scan was used to measure the annulus and aortic root size and to plan the trajectory of the delivery device. The mean annulus size was $24 \pm 3 \mathrm{~mm}$. Our custom-designed self-expanding stent with an expanded diameter of $26 \mathrm{~mm}$ and length of $35 \mathrm{~mm}$ was used for all 8 experiments. There were no issues with stent deployment. The mean time taken to deploy the valve under rtMRI guidance was $61 \pm 5$ seconds.

Postdeployment scans confirmed precise stent placement at $4 \mathrm{~mm}$ below the annulus in all 8 animals. Postdeployment necropsy confirmed the correlation between imaging and the actual valve position (Figure 5). On necropsy, all 8 animals exhibited apposition of the bioprosthesis to the annulus and ascending aorta. All stents were deployed with the proximal end positioned 0-3 mm below the annulus and without occluding the coronary ostia.

\section{DISCUSSION}

In contrast to traditional imaging modalities used for TAVR, rtMRI offers improved soft tissue contrast in addition to a 3-dimensional visualization of anatomic structures. In addition, rtMRI avoids radiation exposure and contrast media. X-ray fluoroscopy exposes the patients and medical staff to significant doses of ionizing radiation, whereas rtMRI uses nonionizing radiation, which has no known deleterious effects to health. ${ }^{31}$ Unlike fluoroscopy, rtMRI guidance does not require the use of contrast media to aid visualization. ${ }^{14-16}$ The use of contrast media can lead to multiple complications, such as contrast-induced nephropathy and anaphylaxis. ${ }^{32}$ Gadolinium can be used

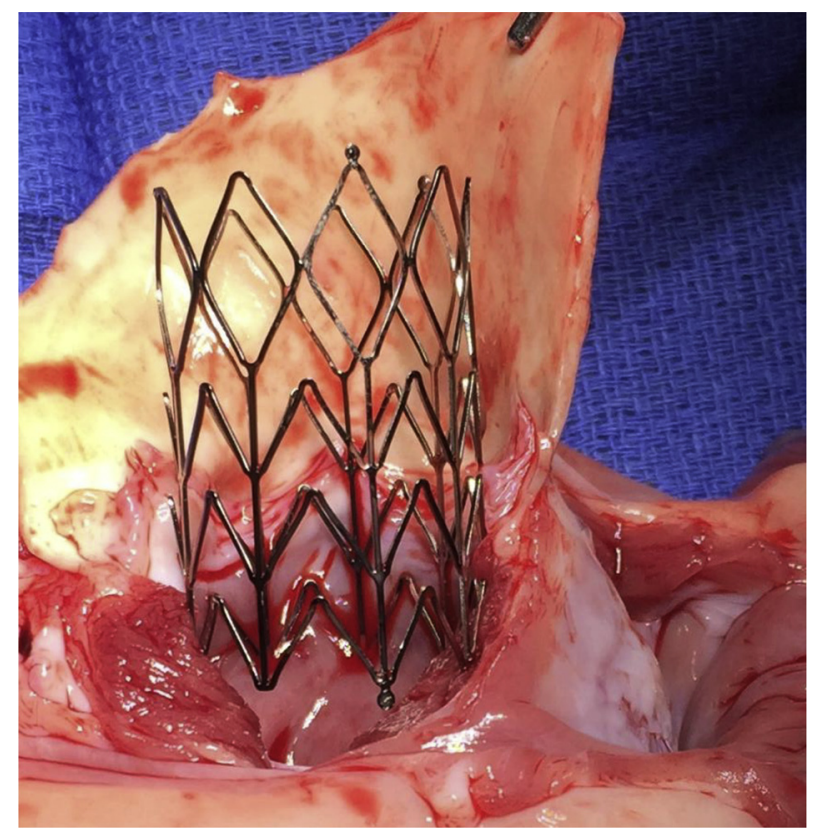

FIGURE 5. Necropsy showing valve placement. 
in a postdeployment, first-pass perfusion to evaluate myocardial perfusion, but it is not required for the procedure. ${ }^{33}$ rtMRI guidance may be useful for patients with renal insufficiency, which is a contraindication to TAVR. ${ }^{17,34}$

Traditional imaging for TAVR requires multiple imaging modalities; however, we have demonstrated in previous experiments that rtMRI can serve as the sole imaging modality for predeployment imaging, imaging guidance for valve deployment, and postdeployment scanning in TAVR procedures with a high accuracy of device delivery and reproducibility. ${ }^{16,21,22,35-38}$ Although rtMRI-guided TAVR is becoming closer to a clinical reality, one of the limitations that we identified during our multiple experiments is the difficulty of performing the procedure in the confines of an MRI suite and working inside the bore of an MRI machine. This revelation led us to develop an MRI-compatible robot-assisted device to aid deployment.

Our experiment demonstrates the feasibility of using this device for transapical rtMRI-guided TAVR; however, there are some limitations to this delivery method, including possible non-MRI-compatible implants, high complexity and cost of the delivery method, device deployment limitations, and the effectiveness of our selfexpanding nitinol stent. Unlike fluoroscopy and CT, the continuous interaction between an MRI's magnetic field with ferromagnetic materials can potentially cause movement and heating of metallic objects, which can lead to tissue damage, as well as damage to electronic circuits. Because of this, patients must be carefully screened before rtMRI procedures. Imaging of patients with non-MRIcompatible implants, such as some implanted cardiac devices, is contraindicated. ${ }^{39,40}$ Patients with non-MRIcompatible implants should be excluded from rtMRIguided TAVR. Another limitation is the complexity and resources required to develop and run a robot-assisted TAVR program. We realize that the costs of hybrid MRI suites and a robotic delivery device would limit its use to only major centers with high-volume TAVR procedures. The device itself also has several limitations. In its current configuration, the robot assistance system is configured for a transapical approach. The device cannot advance a guidewire, and so is not able to perform TAVR from a femoral, subclavian, or axillary access site. Another limitation is stent migration between postdeployment perfusion and necropsy. All 8 stents were deployed precisely at $4 \mathrm{~mm}$ below the annulus, with the position confirmed on postdeployment imaging; however, between postdeployment imaging and necropsy, the stents moved between 1 and $4 \mathrm{~mm}$, owing to the valve design, size mismatch, or valve shifting during harvest for necropsy. Although we are unsure of the exact variable that led to movement, we do know that between deployment and postdeployment imaging, all 8 stents were deployed with millimeter precision.

\section{CONCLUSIONS}

Our results demonstrate the feasibility of robot-assisted TAVR under rtMRI guidance. rtMRI guidance is unique in that this single imaging modality can be successfully used for predeployment, deployment, and postdeployment imaging; however, performing a TAVR while working in the confines of an MRI bore is a challenging task. rtMRI eliminates the deleterious side effects of traditional imaging for TAVR, which includes radiation exposure and contrast media. Our approach using a robotic system may eliminate some of the challenges and may improve the success of TAVR. It provides superior visualization during the insertion process, pinpoint accuracy of deployment, and, potentially, communication between the imaging device and the robotic module to prevent incorrect or misaligned deployment.

\section{Conflict of Interest Statement}

Authors have nothing to disclose with regard to commercial support.

\section{References}

1. Maganti K, Rigolin VH, Sarano ME, Bonow RO. Valvular heart disease diagnosis and management. Mayo Clinic Proc. 2010;85:483-500.

2. Coffey S, Cox B, Williams MJ. The prevalence, incidence, progression, and risks of aortic valve sclerosis: a systematic review and meta-analysis. J Am Coll Cardiol. 2014;63(25 Pt A):2852-61.

3. Nkomo VT, Gardin JM, Skelton TN, Gottdiener JS, Scott CG, EnriquezSarano M. Burden of valvular heart diseases: a population-based study. Lancet 2006;368:1005-11.

4. Carabello BA. Introduction to aortic stenosis. Circ Res. 2013;113:179-85.

5. Ross J Jr, Braunwald E. Aortic stenosis. Circulation. 1968;38:61-7.

6. Turina J, Hess OM, Krayenbühl HP. Spontaneous course of aortic valve disease and indications for aortic valve replacement (in German). Schweiz med Wochenschr. 1988;118:508-16.

7. Leon MB, Smith CR, Mack M, Miller DC, Moses JW, Svensson LG, et al. Transcatheter aortic-valve implantation for aortic stenosis in patients who cannot undergo surgery. N Engl J Med. 2010;363:1597-607.

8. Saeedi M, Thomas A, Shellock FG. Evaluation of MRI issues at 3-Tesla for a transcatheter aortic valve replacement (TAVR) bioprosthesis. Magn Reson Imag ing. 2015;33:497-501

9. Bloomfield GS, Gillam LD, Hahn RT, Kapadia S, Leipsic J, Lerakis S, et al. A practical guide to multimodality imaging of transcatheter aortic valve replacement. JACC Cardiovasc Imaging. 2012;5:441-55.

10. Hahn RT. Use of imaging for procedural guidance during transcatheter aortic valve replacement. Curr Opin Cardiol. 2013;28:512-7.

11. Jilaihawi H, Kashif M, Fontana G, Furugen A, Shiota T, Friede G, et al Cross-sectional computed tomographic assessment improves accuracy of aortic annular sizing for transcatheter aortic valve replacement and reduces the incidence of paravalvular aortic regurgitation. J Am Coll Cardiol. 2012;59: 1275-86.

12. Husser O, Holzamer A, Resch M, Endemann DH, Nunez J, Bodi V, et al Prosthesis sizing for transcatheter aortic valve implantation: comparison of three-dimensional transesophageal echocardiography with multislice computed tomography. Int J Cardiol. 2013;168:3431-8.

13. Smith LA, Dworakowski R, Bhan A, Delithanasis I, Hancock J, Maccarthy PA, et al. Real-time three-dimensional transesophageal echocardiography adds value to transcatheter aortic valve implantation. J Am Soc Echocardiogr. 2013;26: 359-69. 
14. McCullough PA, Wolyn R, Rocher LL, Levin RN, O'Neill WW. Acute renal failure after coronary intervention: incidence, risk factors, and relationship to mortality. Am J Med. 1997;103:368-75.

15. Bruschi G, De Marco F, Martinelli L, Klugmann S. CoreValve ${ }^{\circledR}$ transcatheter self-expandable aortic bioprosthesis. Expert Rev Med Devices. 2013;10:15-26.

16. Horvath KA, Mazilu D, Guttman M, Zetts A, Hunt T, Li M. Midterm results of transapical aortic valve replacement via real-time magnetic resonance imaging guidance. J Thorac Cardiovasc Surg. 2010;139:424-30.

17. Horvath KA, Mazilu D, Cai J, Kindzelski B, Li M. Transapical sutureless aortic valve implantation under magnetic resonance imaging guidance: acute and short-term results. J Thorac Cardiovasc Surg. 2015;149:1067-72.

18. Saeed M, Hetts SW, English J, Wilson M. MR fluoroscopy in vascular and cardiac interventions (review). Int J Cardiovasc Imaging. 2012;28:117-37.

19. Roberts TP, Hassenzahl WV, Hetts SW, Arenson RL. Remote control of catheter tip deflection: an opportunity for interventional MRI. Magn Reson Med. 2002;48: 1091-5.

20. Guttman MA, Kellman P, Dick AJ, Lederman RJ, McVeigh ER. Real-time accelerated interactive MRI with adaptive TSENSE and UNFOLD. Magn Reson Med. 2003;50:315-21.

21. McVeigh ER, Guttman MA, Lederman RJ, Li M, Kocaturk O, Hunt T, et al. Real-time interactive MRI-guided cardiac surgery: aortic valve replacement using a direct apical approach. Magn Reson Med. 2006;56:958-64.

22. Horvath KA, Guttman M, Li M, Lederman RJ, Mazilu D, Kocaturk O, et al. Beating heart aortic valve replacement using real-time MRI guidance, Innovations (Phila). 2007;2:51-5.

23. Horvath KA, Li M, Mazilu D, Guttman MA, McVeigh ER. Real-time magnetic resonance imaging guidance for cardiovascular procedures. Semin Thorac Cardiovasc Surg. 2007; 19:330-5.

24. Lederman RJ. Cardiovascular interventional magnetic resonance imaging. Circulation. 2005;112:3009-17.

25. Horvath KA, Mazilu D, Li M, inventors. Stent for valve replacement. US patent application 61172 568. April 24, 2009.

26. Mazilu D, Li M, Kocaturk O, Horvath KA. Self-expanding stent and delivery system for aortic valve replacement. J Med Device. 2012;6:410061-9.

27. Li M, Mazilu D, Horvath KA. Robotic system for transapical aortic valve replacement with MRI guidance. Med Image Comput Comput Assist Interv. 2008;11(Pt 2):476-84.

28. Li M, Kapoor A, Mazilu D, Horvath KA. Pneumatic actuated robotic assistant system for aortic valve replacement under MRI guidance. IEEE Trans Biomed Eng. 2011;58:443-51.
29. Kapoor A, Wood B, Mazilu D, Horvath KA, Li M. MRI-compatible hands-on cooperative control of a pneumatically actuated robot. IEEE Int Conf Robot Autom. 2009;2009:2681-6.

30. Tsekos NV, Khanicheh A, Christoforou E, Mavroidis C. Magnetic resonance-compatible robotic and mechatronics systems for image-guided interventions and rehabilitation: a review study. Ann Rev Biomed Eng. 2007:9:351-87.

31. Hong J, Wong JT, Bloch KJ. Reactions to radiocontrast media. Allergy Asthma Proc. 2002;23:347-51.

32. Quail MA, Nordmeyer J, Schievano S, Reinthaler M, Mullen MJ, Taylor AM. Use of cardiovascular magnetic resonance imaging for TAVR assessment in patients with bioprosthetic aortic valves: comparison with computed tomography. Eur J Radiol. 2012;81:3912-7.

33. Al-Lamee R, Godino C, Colombo A. Transcatheter aortic valve implantation: current principles of patient and technique selection and future perspectives. Circ Cardiovasc Interv. 2011;4:387-95.

34. Kahlert P, Parohl N, Albert J, Schäfer L, Reinhardt R, Kaiser GM, et al. Towards real-time cardiovascular magnetic resonance guided transarterial CoreValve implantation: in vivo evaluation in swine. J Cardiovasc Magn Reson. 2012;14:21.

35. Kahlert P, Eggebrecht H, Plicht B, Kraff O, McDougall I, Decker B, et al. Towards real-time cardiovascular magnetic resonance-guided transarterial aortic valve implantation: in vitro evaluation and modification of existing devices. $J$ Cardiovasc Magn Reson. 2010;12:58

36. Horvath KA, Mazilu D, Kocaturk O, Li M. Transapical aortic valve replacement under real-time magnetic resonance imaging guidance: experimental results with balloon-expandable and self-expanding stents. Eur J Cardiothorac Surg. 2011; 39:822-8.

37. Kindzelski BA, Li M, Mazilu D, Hunt T, Horvath KA. Real-time magnetic resonance-guided aortic valve replacement using Engager valve. Ann Thorac Surg. 2014;98:2194-9.

38. Kanal E, Borgstede JP, Barkovich AJ, Bell C, Bradley WG, Etheridge S, et al. American College of Radiology White Paper on MR Safety: 2004 update and revisions. AJR Am J Roentgenol. 2004;182:1111-4.

39. Pohost GM. Editor's page: is CMR safe? J Cardiovasc Magn Reson. 2001;3:ix.

40. Sierra M, Machado C. Magnetic resonance imaging in patients with implantable cardiac devices. Rev Cardiovasc Med. 2008;9:232-8.

Key Words: robot-assisted transcatheter aortic valve replacement, real-time magnetic resonance image, swine animal model 